\title{
Correction to: The effect of corticosteroids on the mortality of patients with influenza pneumonia: a systematic review and meta- analysis
}

\author{
Yue-Nan Ni ${ }^{1}$, Guo Chen ${ }^{2}$, Jiankui Sun ${ }^{3}$, Bin-Miao Liang ${ }^{1 *}$ and Zong-An Liang ${ }^{1}$
}

\author{
Correction to: Critical Care (2019) 23:99 \\ https://doi.org/10.1186/s13054-019-2395-8
}

After publication of our article [1], we were made aware of some errors in our figures and tables. There have been no changes to the interpretation of the results, conclusions and applications of our article.

In Fig. 2 and Fig. 3, for Perez-Padilla 2009, the events/total in the corticosteroids and control groups should be $3 / 5$ and $4 / 13$, respectively, instead of $4 / 7$ and $8 / 11$ in the original article. For Viasus 2011, the events/total in the corticosteroids and control groups should be $3 / 37$ and $4 / 160$, respectively, instead of $3 /$ 37 and $7 / 160$ in the original article. Thus, in our final systematic review and meta-analysis, 2,562 patients were treated with corticosteroids and 3,986 with non-corticosteroids. The statistical heterogeneity in the analysis of the effect of corticosteroids on mortality should be $\left(\mathrm{I}^{2}=83 \%, \mathrm{P}<0.00001\right)$, instead of $\left(\mathrm{I}^{2}=84 \%, \mathrm{P}<0.00001\right)$ in the original article. And, the results of the analysis about mortality should be (RR 1.91, 95\% CI 1.42 2.55, $\mathrm{Z}=4.33, \mathrm{P}<0.0001)$, instead of (RR 1.75, 95\% CI 1.30 2.36, $Z=3.71, \mathrm{P}=0.0002$ ). Similarly, the results of the subgroup mortality in H1N1 patients should be (RR 1.92, 95\% CI

\footnotetext{
The original article can be found online at https://doi.org/10.1186/s13054019-2395-8.

* Correspondence: liangbinmiao@163.com

${ }^{1}$ Department of Respiratory and Critical Care Medicine, West China School of Medicine and West China Hospital, Sichuan University, No.37 Guoxue Alley, Chengdu 610041, Sichuan, China

Full list of author information is available at the end of the article
}

1.23 3.02, $\mathrm{Z}=2.85, \mathrm{P}=0.004)$, rather than ( $\mathrm{RR} 1.69$, 95\% CI 1.15 2.47, $\mathrm{Z}=2.68, \mathrm{P}=0.007$ ).

Otherwise, Lee 2014 should be Lee 2015.

In Fig. 5, the SD for the corticosteroids and control groups in Brun-Buisson's study should be 19.26 and 14.07, respectively, instead of 14.07 and 19.26 in the original article. Thus, the statistical heterogeneity of the analysis on ICU LOS should be $\left(\mathrm{I}^{2}=\right.$ $30 \%, \mathrm{P}=0.23)$, instead of $\left(\mathrm{I}^{2}=38 \%, \mathrm{P}=0.21\right)$. And the result of this analysis should be (MD 2.12, 95\% CI 1.15 3.09, $\mathrm{Z}=4.30, \quad \mathrm{P}<0.0001$ ), rather than (MD $2.14,95 \%$ CI $1.17 \sim 3.10, \mathrm{Z}=4.35, \mathrm{P}<0.0001)$ in the original article.

In Fig. 6, the study ID "Dias 2012" should be "Viasus 2011".

In table 1, the antiviral drug in Lee's study should be NR.

In table 2, for Brun-Buisson's study, the Male (n, $\%)$ in the corticosteroids group should be 36(43.4), and 69(55.2) in the control group. For Moreno's study, the APACHE II in the corticosteroids group should be 15(10-20) and 14(10-19) in the control group.

And, the age and male in the control group of Viasus's study should be NR. 


\begin{tabular}{|c|c|c|c|c|c|c|c|c|c|}
\hline \multirow[b]{2}{*}{ Studv or Subgroup } & \multicolumn{2}{|c|}{ Corticosteroids } & \multicolumn{2}{|c|}{ Control } & \multirow{2}{*}{ Weight } & \multirow{2}{*}{$\begin{array}{c}\text { Risk Ratio } \\
\text { M-H. Random, } 95 \% \mathrm{Cl}\end{array}$} & \multirow{2}{*}{\multicolumn{3}{|c|}{$\begin{array}{c}\text { Risk Ratio } \\
\text { M-H, Random, } 95 \% \mathrm{Cl}\end{array}$}} \\
\hline & Events & Total & Events & Total & & & & & \\
\hline Diaz 2012 & 25 & 136 & 41 & 236 & $10.6 \%$ & $1.06[0.67,1.66]$ & & & \\
\hline Rios 2011 & 38 & 75 & 47 & 103 & $12.3 \%$ & $1.11[0.82,1.51]$ & & - & \\
\hline Moreno 2018 & 166 & 604 & 234 & 1242 & $13.5 \%$ & $1.46[1.23,1.74]$ & & $\rightarrow$ & \\
\hline Lee 2015 & 50 & 264 & 87 & 817 & $12.2 \%$ & $1.78[1.29,2.45]$ & & & \\
\hline Perez-Padilla 2009 & 3 & 5 & 4 & 13 & $4.8 \%$ & $1.95[0.66,5.77]$ & & & \\
\hline Brun-Buisson 2011 & 28 & 83 & 21 & 125 & $10.1 \%$ & $2.01[1.23,3.29]$ & & & \\
\hline Jung 2011 & 54 & 99 & 24 & 120 & $11.2 \%$ & $2.73[1.83,4.07]$ & & & \\
\hline Cao 2016 & 81 & 204 & 11 & 84 & $9.2 \%$ & $3.03[1.70,5.40]$ & & & \\
\hline Li 2017 & 232 & 1055 & 74 & 1086 & $12.9 \%$ & $3.23[2.52,4.13]$ & & $\because$ & \\
\hline Viasus 2011 & 3 & 37 & 4 & 160 & $3.1 \%$ & $3.24[0.76,13.88]$ & & & \\
\hline Total $(95 \% \mathrm{Cl})$ & & 2562 & & 3986 & $100.0 \%$ & $1.91[1.42,2.55]$ & & & \\
\hline Total events & 680 & & 547 & & & & & & \\
\hline $\begin{array}{l}\text { Heterogeneity: } \mathrm{Tau}^{2}= \\
\text { Test for overall effect: }\end{array}$ & $\begin{array}{l}0.16 ; \mathrm{Chi}^{2}= \\
\mathrm{Z}=4.33(\mathrm{P}\end{array}$ & $\begin{array}{l}51.54, \mathrm{df} \\
0.0001)\end{array}$ & $=9\langle P<$ & 0.0000 & $01) ; I^{2}=83$ & & $\begin{array}{cccc}1 & 1 & 1 \\
0.1 & 0.2 & 0.5 & 1 \\
& \text { Favours corticosteroids }\end{array}$ & $\begin{array}{cc}2 & 5 \\
\text { Favours control }\end{array}$ & 10 \\
\hline
\end{tabular}

\begin{tabular}{|c|c|c|c|c|c|c|c|c|c|}
\hline \multirow[b]{2}{*}{ Studv or Subgroup } & \multicolumn{2}{|c|}{ Corticosteroids } & \multicolumn{2}{|c|}{ Control } & & Risk Ratio & \multirow{2}{*}{\multicolumn{3}{|c|}{$\begin{array}{c}\text { Risk Ratio } \\
\text { M-H. Random. } 95 \% \mathrm{Cl}\end{array}$}} \\
\hline & Events & Total & Events & Total & Weight & M-H. Random, $95 \% \mathrm{Cl}$ & & & \\
\hline Brun-Buisson 2011 & 28 & 83 & 21 & 125 & $15.7 \%$ & $2.01[1.23,3.29]$ & & & \\
\hline Diaz 2012 & 25 & 136 & 41 & 236 & $16.2 \%$ & $1.06[0.67,1.66]$ & & & \\
\hline Jung 2011 & 54 & 99 & 24 & 120 & $16.7 \%$ & $2.73[1.83,4.07]$ & & $\rightarrow$ & \\
\hline Li 2017 & 232 & 1055 & 74 & 1086 & $18.2 \%$ & $3.23[2.52,4.13]$ & & $\rightarrow-$ & \\
\hline Perez-Padilla 2009 & 3 & 5 & 4 & 13 & $9.1 \%$ & $1.95[0.66,5.77]$ & & & \\
\hline Rios 2011 & 38 & 75 & 47 & 103 & $17.7 \%$ & $1.11[0.82,1.51]$ & & - & \\
\hline Viasus 2011 & 3 & 37 & 4 & 160 & $6.4 \%$ & $3.24[0.76,13.88]$ & & & \\
\hline Total $(95 \% \mathrm{Cl})$ & & 1490 & & 1843 & $100.0 \%$ & $1.92[1.23,3.02]$ & & & \\
\hline Total events & 383 & & 215 & & & & & & \\
\hline $\begin{array}{l}\text { Heterogeneity: } \mathrm{Tau}^{2} \\
\text { Test for overall effect }\end{array}$ & $\begin{array}{l}0.27 ; \mathrm{Chi}^{2}= \\
\mathrm{z}=2.85(\mathrm{P}\end{array}$ & $\begin{array}{l}40.45, \mathrm{df} \\
0.004)\end{array}$ & $=6(P<$ & $<0.0000$ & $01) ; 1^{2}=85$ & & $\begin{array}{ccc}0.1 & 0.2 & 0.5\end{array}$ & $\begin{array}{lcr}1 & 2 & 5 \\
& 2 & \end{array}$ & 10 \\
\hline
\end{tabular}

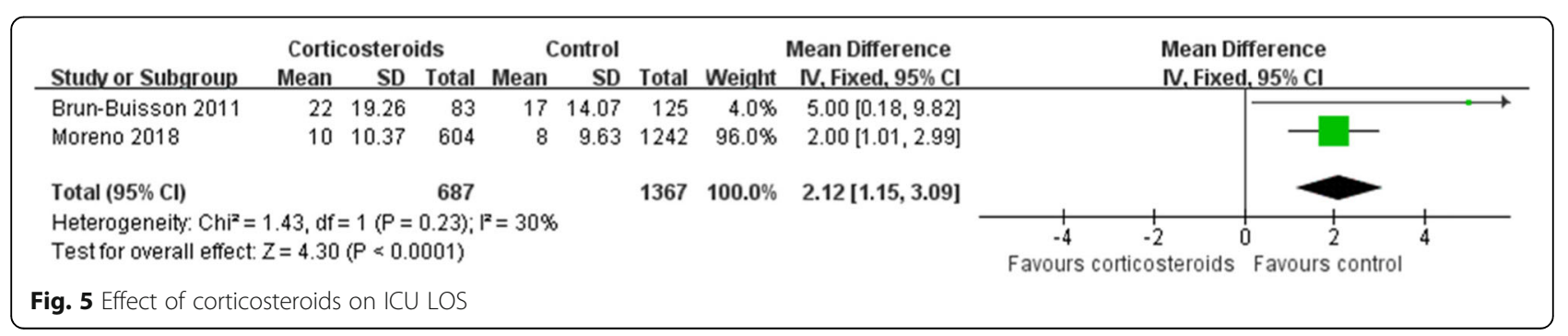




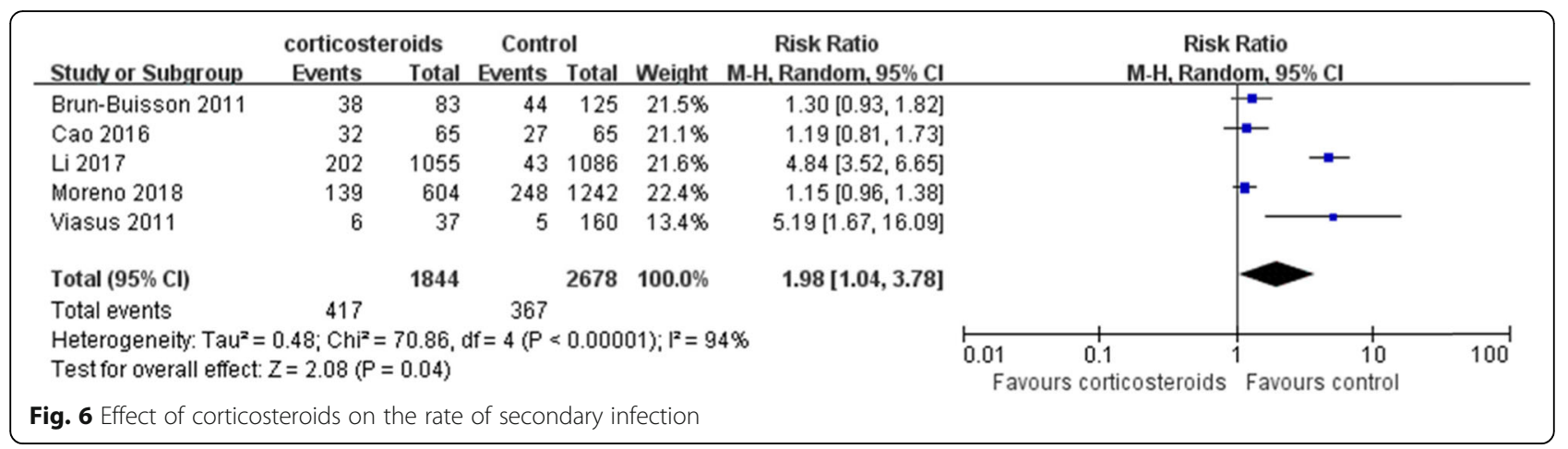

\section{Author details}

'Department of Respiratory and Critical Care Medicine, West China School of Medicine and West China Hospital, Sichuan University, No.37 Guoxue Alley, Chengdu 610041, Sichuan, China. ${ }^{2}$ Department of Geriatrics, Sichuan Academy of Medical Sciences \& Sichuan Provincial People's Hospital, Chengdu, Sichuan, China. ${ }^{3}$ State Key Laboratory of Oral Diseases, West China School of Stomatology, Sichuan University, No.14, Section 3 Renmin Nanlu, Chengdu 610041, Sichuan, China.

Published online: 23 June 2020

\section{Reference}

1. Ni YN, Chen G, Sun J, Liang BM, Liang ZA. The effect of corticosteroids on mortality of patients with influenza pneumonia: a systematic review and meta-analysis. Critical Care (London, England). 2019;23:99. https://doi.org/10. 1186/s13054-019-2395-8. 